Pacific

Journal of

Mathematics

NONSYMPLECTIC 4-MANIFOLDS WITH ONE BASIC CLASS

Ronald Fintushel and Ronald J. Stern 


\title{
NONSYMPLECTIC 4-MANIFOLDS WITH ONE BASIC CLASS
}

\author{
Ronald Fintushel And Ronald J. Stern
}

We produce examples of simply connected 4-manifolds which have (up to sign) only one class with a nontrivial Seiberg-Witten invariant. Furthermore, these manifolds admit no symplectic structure with either orientation.

\section{Introduction.}

In the past few years Zoltan Szabo $[\mathbf{S 1}, \mathbf{S 2}]$ and the authors [FS2] have produced examples of simply connected irreducible 4-manifolds which do not admit a symplectic structure with either orientation. We shall call such manifolds nonsymplectic. Due to the nature of their construction, these manifolds have many basic classes. It is the purpose of this paper to construct families of examples of nonsymplectic 4-manifolds which (up to sign) have just one basic class.

The key to detecting that the manifolds of $[\mathbf{S 1}, \mathbf{S 2}]$ and of $[\mathbf{F S 2}]$ are not symplectic lies in the theorem of $\mathrm{C}$. Taubes which states that the SeibergWitten invariant associated to the canonical class of a symplectic 4-manifold is \pm 1 . Recall that the Seiberg-Witten invariant $\mathrm{SW}_{X}$ of a smooth closed oriented 4-manifold $X$ with $b^{+}>1$ is an integer valued function which is defined on the set of $\operatorname{spin}^{c}$ structures over $X$ (cf. $\left.[\mathbf{W}]\right)$. In case $H_{1}(X ; \mathbf{Z})$ has no 2-torsion, there is a natural identification of the $\operatorname{spin}^{c}$ structures of $^{2}$ $X$ with the characteristic elements of $H^{2}(X ; \mathbf{Z})$. In this case we view the Seiberg-Witten invariant as

$$
\mathrm{SW}_{X}:\left\{k \in H^{2}(X, \mathbf{Z}) \mid k \equiv w_{2}(T X) \quad(\bmod 2)\right\} \rightarrow \mathbf{Z} .
$$

The Seiberg-Witten invariant $\mathrm{SW}_{X}$ is a smooth invariant whose sign depends on an orientation of $H^{0}(X ; \mathbf{R}) \otimes \operatorname{det} H_{+}^{2}(X ; \mathbf{R}) \otimes \operatorname{det} H^{1}(X ; \mathbf{R})$. If $\mathrm{SW}_{X}(\beta) \neq$ 0 , then $\beta$ is called a basic class of $X$. It is a fundamental fact that the set of basic classes is finite. If $\beta$ is a basic class, then so is $-\beta$ with

$$
\mathrm{SW}_{X}(-\beta)=(-1)^{(\mathrm{e}+\operatorname{sign})(X) / 4} \mathrm{SW}_{X}(\beta)
$$

where $\mathrm{e}(X)$ is the Euler number and $\operatorname{sign}(X)$ is the signature of $X$. Because of this, we shall say that $X$ has $n$ basic classes if the set $\left\{\beta \mid \operatorname{SW}_{X}(\beta) \neq\right.$ $0\} /\{ \pm 1\}$ consists of $n$ elements. 
There are abundant examples of 4-manifolds with one basic class. Minimal nonsingular algebraic surfaces of general type have one basic class (the canonical class) $[\mathbf{W}]$. The authors have constructed many examples of minimal symplectic manifolds with one basic class and $\chi-3 \leq c_{1}{ }^{2}<2 \chi-6$, where $\chi=\left(b^{+}+1\right) / 2$. (These manifolds cannot admit complex structures due to the geography of complex surfaces.) However, the examples produced below are the first nonsymplectic manifolds with one basic class.

As in [FS2] we need to view the Seiberg-Witten invariant as a Laurent polynomial. To do this, let $\left\{ \pm \beta_{1}, \ldots, \pm \beta_{n}\right\}$ be the set of nonzero basic classes for $X$. We may then view the Seiberg-Witten invariant of $X$ as the 'symmetric' Laurent polynomial

$$
\mathcal{S} \mathcal{W}_{X}=b_{0}+\sum_{j=1}^{n} b_{j}\left(t_{j}+(-1)^{(\mathrm{e}+\operatorname{sign})(X) / 4} t_{j}^{-1}\right)
$$

where $b_{0}=\mathrm{SW}_{X}(0), b_{j}=\mathrm{SW}_{X}\left(\beta_{j}\right)$ and $t_{j}=\exp \left(\beta_{j}\right)$. The examples of $[\mathbf{S 1}, \mathbf{S 2}]$ and of [FS2] are obtained by producing 4-manifolds whose SeibergWitten Laurent polynomial $\mathcal{S} \mathcal{W}_{X}$ has as a factor a nonmonic (symmetrized) Alexander polynomial of a knot or link. Taubes' result is then used to show that $X$ cannot have a symplectic structure. It is not difficult to see that any nonsymplectic manifold (with $b^{+}>1$ ) which can be constructed by the techniques of [FS2] (as explained in [FS2], this includes the examples of Szabo) must have more than one basic class.

Whereas the examples of [FS2] are constructed by surgeries on embedded tori of self-intersection 0, the examples presented here arise from surgeries on higher genus surfaces. These examples are described in the next section.

\section{A new family of 4-manifolds.}

We begin by recalling the construction of [FS2]. Suppose that we are given a smooth simply connected oriented 4-manifold $X$ containing an essential smoothly embedded torus $T$ of self-intersection 0 . Suppose further that $\pi_{1}(X \backslash T)=1$ and that $T$ is contained in a cusp neighborhood. Let $K \subset S^{3}$ be a smooth knot and $M_{K}$ the 3-manifold obtained from 0 -framed surgery on $K$. The meridional loop $m$ to $K$ defines a 1-dimensional homology class $[m]$ both in $S^{3} \backslash K$ and in $M_{K}$. Denote by $T_{m}$ the torus $S^{1} \times m \subset S^{1} \times M_{K}$. Then $X_{K}$ is defined to be the fiber sum

$$
X_{K}=X \#_{T=T_{m}} S^{1} \times M_{K}=(X \backslash N(T)) \cup\left(S^{1} \times\left(S^{3} \backslash N(K)\right)\right),
$$

where $N(T) \cong D^{2} \times T^{2}$ is a tubular neighborhood of $T$ in $X$ and $N(K)$ is a neighborhood of $K$ in $S^{3}$. If $\lambda$ denotes the longitude of $K$ ( $\lambda$ bounds a surface in $\left.S^{3} \backslash K\right)$ then the gluing of this fiber sum identifies $\{\mathrm{pt}\} \times \lambda$ with a normal circle to $T$ in $X$. The main theorem of [FS2] asserts that $X_{K}$ is 
homeomorphic to $X$, and

$$
\mathcal{S} \mathcal{W}_{X_{K}}=\mathcal{S} \mathcal{W}_{X} \cdot \Delta_{K}(t)
$$

where $\Delta_{K}$ is the symmetrized Alexander polynomial of $K$ and $t=\exp (2[T])$.

To begin our construction, take $X$ to be the $K 3$-surface (which has $\mathcal{S} \mathcal{W}_{X}=1$ ) and let the torus $T$ be a smooth fiber of an elliptic fibration on $X$. The pair $(X, T)$ satisfies the hypotheses of [FS2]; so for any knot $K$ we get a homotopy $K 3$-surface $X_{K}$ whose Seiberg-Witten invariant is $\mathcal{S} \mathcal{W}_{X_{K}}=\Delta_{K}(t)$. The $K 3$-surface, $X$, has a section (to the elliptic fibration) which is a smoothly embedded 2-sphere $S$ of self-intersection -2 , and $[S] \cdot[T]=1$. The sum $[S]+[T]$ is represented by a smooth torus $\Sigma$ with $[\Sigma]^{2}=0$ and $[\Sigma] \cdot[T]=1$.

Suppose that the knot $K$ has genus $g$. In the construction of $X_{K}$ we have replaced a 2-disk in $S$ (normal to $T$ ) with a punctured surface of genus g. Thus $X_{K}$ contains a genus $g$ surface $S^{\prime}$ satisfying $\left[S^{\prime}\right] \cdot\left[S^{\prime}\right]=-2$ and $\left[S^{\prime}\right] \cdot[T]=1$. Consider another smooth fiber $T^{\prime}$ of the elliptic fibration of $(X \backslash N(T)) \subset X_{K}$. Then $T^{\prime}+S^{\prime}$ is a singular curve with one double point, which can be smoothed to give an embedded surface $\Sigma^{\prime}$ of genus $g+1$ representing the homology class $\left[\Sigma^{\prime}\right]=\left[T^{\prime}\right]+\left[S^{\prime}\right]$. Thus $\left[\Sigma^{\prime}\right]^{2}=0$ and $\left[\Sigma^{\prime}\right] \cdot[T]=1$.

Next, let $K^{\prime}$ denote the left-handed trefoil knot in $S^{3}$. Since $K^{\prime}$ is a fibered genus 1 knot, the 4-manifold $S^{1} \times M_{K^{\prime}}$ is a smooth $T^{2}$-fiber bundle over $T^{2}$. Forming the fiber sum of $g+1$ copies of $S^{1} \times M_{K^{\prime}}$, we obtain

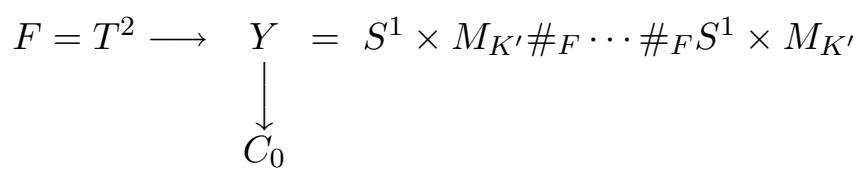

where $C_{0}$ is a genus $g+1$ surface. Furthermore, $S^{1} \times M_{K^{\prime}}$ is a symplectic manifold $[\mathbf{T h}]$. Notice that there is a section $C \subset Y$ of the fibration given by the connected sum of the $g+1$ tori $T_{m_{i}}$.

Generally, suppose that we are given symplectic 4 -manifolds $A$ and $B$ and that $A \#_{N} B$ is their symplectic fiber sum along a symplectic torus of selfintersection 0 . The adjunction formula implies that the canonical class $K_{A}$ is orthogonal to [N], as is $K_{B}$. The canonical class of $A \#_{N} B$ is then $K_{A \#_{N} B}=$ $K_{A}+K_{B}+2[N]$ (cf. [G]). Apply this fact to $X_{K^{\prime}}=K 3 \#_{T=T_{m}} S^{1} \times M_{K^{\prime}}$. Since $\mathcal{S} \mathcal{W}_{X_{K^{\prime}}}=\Delta_{K^{\prime}}(t)=t-1+t^{-1}$ (where $t=\exp \left(2\left[T_{m}\right]\right)$ ), we see that $K_{X_{K^{\prime}}}=2 T_{m}=K_{K 3}+K_{S^{1} \times M_{K^{\prime}}}+2\left[T_{m}\right]=K_{S^{1} \times M_{K^{\prime}}}+2\left[T_{m}\right]$. Hence $K_{S^{1} \times M_{K^{\prime}}}=0$ and so $c_{1}\left(K_{S^{1} \times M_{K^{\prime}}}\right)=0$. Now apply the fact $g$ more times, this time fiber-summing along $F$. It follows that $Y$ is a symplectic 4-manifold with $c_{1}(Y)=-2 g[F]$. (Here, we identify $[F]$ with its Poincaré dual.)

Our example, corresponding to the genus $g$ knot $K$ is

$$
Z_{K}=X_{K} \#_{\Sigma^{\prime}=C} Y \text {. }
$$


We perform this fiber sum so that $Z_{K}$ is a spin 4-manifold [G].

Proposition 2.1. The manifold $Z_{K}$ is simply connected.

Proof. The fundamental group $\pi_{1}\left(X_{K} \backslash \Sigma^{\prime}\right)$ is normally generated by a boundary circle of a normal disk to $\Sigma^{\prime}$. Since $\left[\Sigma^{\prime}\right] \cdot[T]=1$, we may assume that this circle lies on a copy $\{\mathrm{pt}\} \times T$ in the boundary $\partial D^{2} \times T=\partial N(T)$. We claim that there are generators $\lambda_{1}, \lambda_{2}$, for $\pi_{1}(T)$ which bound vanishing cycles (disks of self-intersection -1) in $X \backslash\left(S \cup T^{\prime}\right)$. (Note that here we are identifying $X \backslash T$ with $X_{K} \backslash T$.) This claim can be seen to be true inside a $K 3$ nucleus, i.e., in a regular neighborhood of the union of $S$ and a cusp fiber. A Kirby calculus diagram for the nucleus is given in the Figure 1 below.

The section $S$ is the union of the disk spanned by the circle labelled ' -2 ' and the core of the 2-handle which is attached to it. The torus $T$ is obtained as follows. The circle labelled ' 0 ' bounds a disk $D$ which is punctured in two points by one of the dotted circles. Remove a pair of disks from $D$ at these intersection points, and connect the boundaries of these disks with an annulus which surrounds the path $\gamma$ in the diagram. The torus $T$ is the union of the twice-punctured $D$ and this annulus. We can see that the loop $\alpha$ of the diagram lies on $T$, and it is easy to deform $\beta$ to also lie on $T$. (When this is done, $\alpha$ and $\beta$ will intersect in a point.) Thus $H_{1}(T)$ is generated by the classes represented by the loops $\alpha$ and $\beta$. The vanishing cycles are the cores of the $(-1)$-framed 2-handles which are attached to $\alpha$ and $\beta$. This proves the claim.

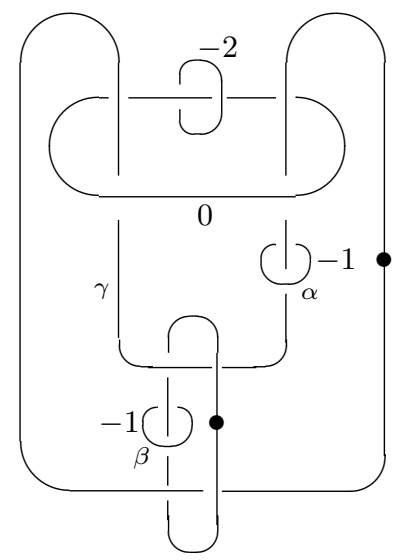

Figure 1.

This means that $\pi_{1}(\{\mathrm{pt}\} \times T) \rightarrow \pi_{1}(X \backslash \Sigma)$ is the zero map; hence $\pi_{1}(\{\mathrm{pt}\} \times T) \rightarrow \pi_{1}\left(X_{K} \backslash \Sigma^{\prime}\right)$ is also the zero map. However, $\pi_{1}\left(X_{K} \backslash \Sigma^{\prime}\right)$ is normally generated by the image of this map; so $X_{K} \backslash \Sigma^{\prime}$ is simply connected. 
Thus

$$
\pi_{1}\left(Z_{K}\right)=\pi_{1}\left(X_{K} \backslash \Sigma^{\prime}\right) *_{1}\left(C \times S^{1}\right) \pi_{1}(Y \backslash C)=\pi_{1}(Y \backslash C) / \pi_{1}\left(C \times S^{1}\right) .
$$

Because $\pi_{1}\left(S^{3} \backslash K^{\prime}\right)$ is normally generated by the meridian $m, \pi_{1}\left(S^{1} \times\right.$ $\left.\left(S^{3} \backslash K^{\prime}\right)\right)=\pi_{1}\left(\left(S^{1} \times M_{K^{\prime}}\right) F\right)$ is normally generated by $\pi_{1}\left(S^{1} \times m\right)$. An inductive application of Van Kampen's theorem shows that $\pi_{1}(Y)$ is normally generated by $\pi_{1}\left(S^{1} \times m \# \cdots \# S^{1} \times m\right)=\pi_{1}(C)$. Thus $\pi_{1}(Y \backslash C)$ is normally generated by $\pi_{1}\left(C \times S^{1}\right)$, and so $\pi_{1}\left(Z_{K}\right)=1$.

\section{The Seiberg-Witten invariants of $Z_{K}$.}

Consider first $H_{2}\left(Z_{K}\right)$. There is an important class $\tau \in H_{2}\left(Z_{K}\right)$ constructed as follows. In the construction of $Z_{K}$, the boundary of a tubular neighborhood $N\left(\Sigma^{\prime}\right)$ of $\Sigma^{\prime}$ in $X_{K}$ is identified with the boundary of a tubular neighborhood $N(C)$ of $C$ in $Y$. Fix a fiber $F$ of $Y$, and let $F_{0}=F \backslash(F \cap \operatorname{int} N(C))$. There is torus $T^{\prime \prime}$ which is a smooth fiber of the elliptic fibration of $X \backslash\left\{T \cup T^{\prime}\right\} \subset X_{K}$ and such that if $T_{0}^{\prime \prime}=T^{\prime \prime} \backslash\left(T^{\prime \prime} \cap \operatorname{int} N\left(\Sigma^{\prime}\right)\right)$, then $\partial T_{0}^{\prime \prime}=\partial F_{0}$ in $Z_{K}$. Let $\tau$ denote the class $\tau=\left[T_{0}^{\prime \prime} \cup F_{0}\right]$. Note that $\tau$ is represented by an embedded surface of genus $2, \tau^{2}=0$, and $\tau \cdot\left[\Sigma^{\prime}\right]=1$. Then $H_{2}\left(Z_{K}\right)$ is generated by the image of $H_{2}(Y \backslash C)$, of $H_{2}\left(X_{K} \backslash \Sigma^{\prime}\right)$, and the class $\tau$. The only other classes which could contribute to $H_{2}\left(Z_{K}\right)$ are the classes of rim tori, i.e., tori lying on $\partial N\left(\Sigma^{\prime}\right)=\partial N(C)$ in $Z_{K}$ which have the form $\xi \times \partial D^{2}$ where $D^{2}$ is a normal disk to $\Sigma^{\prime}$ (or to $C$ ). The next lemma shows that in fact they are all trivial.

Lemma 3.1. Each rim torus is homologically trivial in $Z_{K}$.

Proof. A rim torus on $\partial N(C)$ has the form $\xi \times \partial D^{2}$, for some loop $\xi$ on $C$. Recall that there is a fiber bundle $\varphi: Y \rightarrow C_{0}$ with fiber $F$. Let $Q=\varphi^{-1}(\xi) \subset Y$. We see that $\xi \times \partial D^{2}$ bounds the 3-chain $Q \backslash\left(\xi \times \operatorname{int} D^{2}\right)$ in $Y \backslash N(C) \subset Z_{K}$.

Before we prove our main theorem, we recall that a 4 -manifold $W$ is said to have simple type if $\mathrm{SW}_{W}(k) \neq 0$ implies that

$$
\operatorname{dim} \mathcal{M}_{W}(k)=\frac{1}{4}\left(k^{2}-(3 \operatorname{sign}+2 \mathrm{e})(W)\right)=0
$$

where $\mathcal{M}_{W}(k)$ is the Seiberg-Witten moduli space. Write the symmetrized Alexander polynomial of $K$ as $\Delta_{K}(t)=a_{0}+\sum_{n=1}^{d} a_{n}\left(t^{n}+t^{-n}\right)$. We call $d$ the degree of $\Delta_{K}(t)$. We are assuming that the genus of $K$ is $g$; so $d \leq g$. If $K$ is an alternating knot, for example, then $d=g$. Let us say that the Alexander polynomial of $K$ has maximal degree if $d=g$.

Theorem 3.2. Let $K$ be a knot in $S^{3}$ whose Alexander polynomial has maximal degree. Then $Z_{K}$ is of simple type and has (up to sign) a single basic 
class, $k=2 g \tau+2\left[\Sigma^{\prime}\right]$. Furthermore, $\left|\mathrm{SW}_{Z_{K}}(k)\right|=a_{d}$, the top coefficient of $\Delta_{K}(t)$.

Proof. Let $U$ denote a nucleus in $X=K 3$ which contains the fiber $T$ and section $S$ from the construction of $X_{K}$. We see that $(X \backslash U) \subset\left(X_{K} \backslash \Sigma^{\prime}\right)$. The homology $H_{2}(X \backslash U) \cong 2 E_{8} \oplus 2 H$, where the negative definite $E_{8}$ forms are generated by the classes of embedded spheres of self-intersection -2 , and the two hyperbolic pairs $H$ are each generated by a torus $T_{i}$ of selfintersection 0 , and a sphere $S_{i}$ of self-intersection -2 which meet transversely in a single point. The homology $H_{2}\left(X_{K} \backslash \Sigma^{\prime}\right)$ is generated by the image of $H_{2}(X \backslash U)$ together with $\left[\Sigma^{\prime}\right]$ and the classes of rim tori.

Next consider $Y=S^{1} \times M_{K^{\prime}} \#_{F} \cdots \#_{F} S^{1} \times M_{K^{\prime}}(g+1$ copies $)$ where $F$ is the torus fiber of the fibration of $S^{1} \times M_{K^{\prime}}$ over the torus. Each $S^{1} \times M_{K^{\prime}}$ has the homology of $S^{2} \times T^{2}$. Each fiber sum in the construction of $Y$ increases the first betti number $b_{1}$ by 2 - the base of the fibration has genus increased by $1-$ and $H_{2}$ is increased by the addition of two hyperbolic pairs as follows: Choose a standard basis $\left\{x_{1}, x_{2}\right\}$ for $H_{1}(F ; \mathbf{Z})$. For example, $x_{1}$ is represented by a loop as shown in Figure 2. Each of the curves $x_{i}$ bounds a punctured torus $\Gamma_{i}$ in $M_{K}$. In Figure $3, x_{1}$ is isotopic to the pictured curve $x_{1}^{\prime}$, and the punctured torus is composed of the twice-punctured disk which $x_{1}^{\prime}$ bounds, together with a 1-handle which pipes around the knot. Let $x_{i}^{\prime \prime}$ be a push-off of $x_{i}$ in $F$. Then the linking number of $x_{i}$ and $x_{i}^{\prime \prime}$ is +1 . (Here we are using the fact that $K^{\prime}$ is a left-hand trefoil knot.) This means that the self-intersection number of $\Gamma_{i}$ (as a surface in $M_{K} \times I$, say), keeping its boundary in $F$, is +1 .

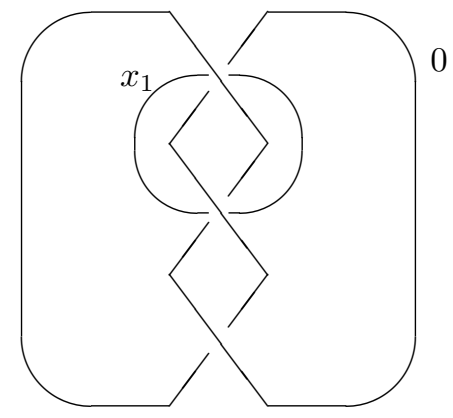

Figure 2.

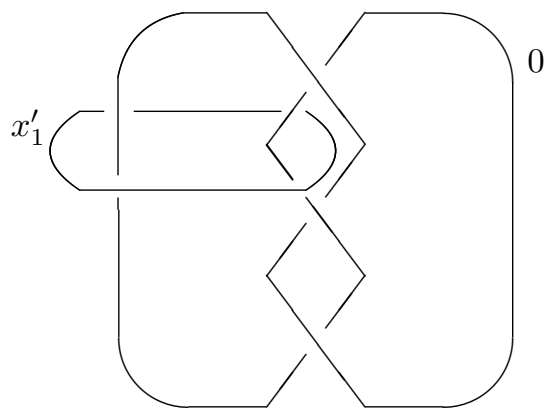

Figure 3.

Thus in $S^{1} \times M_{K^{\prime}} \#{ }_{F} S^{1} \times M_{K^{\prime}}$ one produces genus 2 surfaces $S_{1}^{\prime}, S_{2}^{\prime}$, of self-intersection +2 which are formed from pairs of these tori. Let $T_{1}^{\prime}$, $T_{2}^{\prime}$ be the rim tori corresponding to $x_{2}, x_{1}$ (reversed on purpose). Then in $H_{2}\left(S^{1} \times M_{K^{\prime}} \#_{F} S^{1} \times M_{K^{\prime}} ; \mathbf{Z}\right)$ two hyperbolic pairs are generated by the pairs $\left\{\left[S_{i}^{\prime}\right],\left[T_{i}^{\prime}\right]\right\}$. Each further fiber sum adds two such hyperbolic pairs to 
$H_{2}$. It follows that $H_{2}(Y \backslash C)$, is generated by the $\left[S_{i}^{\prime}\right],\left[T_{i}^{\prime}\right]$, and the section class $[C]$.

Using our observations above, if $k$ is a basic class of $Z_{K}$ we can write

$$
k=a \tau+b\left[\Sigma^{\prime}\right]+\beta+\sum_{i=1}^{2} m_{i}\left[T_{i}\right]+n_{i}\left[S_{i}\right]+\sum_{j=1}^{2 g} t_{j}\left[T_{j}^{\prime}\right]+s_{j}\left[S_{j}^{\prime}\right]
$$

where $a>0$ and $\beta \in 2 E_{8} \subset H_{2}(X \backslash U)$. The adjunction inequality (see e.g. [MST]) states that if $k$ is a basic class and $B$ is an embedded surface of genus $g_{B}$ and self-intersection $[B]^{2} \geq 0$ then

$$
2 g_{B}-2 \geq[B]^{2}+|k \cdot[B]| \cdot
$$

In particular, this implies that if the self-intersection of $B$ is $[B]^{2}=2 g_{B}-2$, then any basic class $k$ must be orthogonal to it: $k \cdot[B]=0$. Since $T_{i}$ is a torus of self-interesection 0 , it follows that $n_{i}=k \cdot\left[T_{i}\right]=0$, and, since $\left[S_{i}\right]+\left[T_{i}\right]$ is also represented by an embedded torus of self-intersection 0 , $m_{i}+n_{i}=k \cdot\left(\left[T_{i}\right]+\left[S_{i}\right]\right)=0$. The same argument applies to show that $s_{j}=0=t_{j}$ for each $j=1, \ldots, 2 g$. Thus $k=a \tau+b\left[\Sigma^{\prime}\right]+\beta$. Apply the adjunction inequality to the genus $g+1$ surface $\Sigma^{\prime}$ and the genus 2 surface representing $\tau$ to obtain:

$$
a=k \cdot\left[\Sigma^{\prime}\right] \leq 2 g, \quad|b|=|k \cdot \tau| \leq 2 .
$$

Because $k$ is a basic class, $\operatorname{dim} \mathcal{M}_{Z_{K}}(k) \geq 0$, hence

$$
0 \leq k^{2}-(3 \operatorname{sign}+2 \mathrm{e})\left(Z_{K}\right) .
$$

Since $(3 \operatorname{sign}+2 \mathrm{e})\left(X_{K}\right)=0$, and $(3 \operatorname{sign}+2 \mathrm{e})(Y)=0$, it is easy to check that $(3 \operatorname{sign}+2 \mathrm{e})\left(Z_{K}\right)=8 g$. Furthermore, $\beta$ lies in the negative definite space $2 E_{8}$; so if $\beta \neq 0$ then

$$
0 \leq 2 a b+\beta^{2}-8 g<2 a b-8 g \leq 8 g-8 g=0 .
$$

This contradiction implies that $\beta=0$; so $k=a \tau+b\left[\Sigma^{\prime}\right]$. Any of the $(-2)$ spheres generating the $E_{8}$ 's is orthogonal to $k$; hence orthogonal to each basic class of $Z_{K}$. It now follows from [FS1] that $Z_{K}$ has simple type; $\operatorname{dim} \mathcal{M}_{Z_{K}}(k)=0$. Thus we have

$$
2 a b=k^{2}=(3 \operatorname{sign}+2 \mathrm{e})\left(Z_{K}\right)=8 g .
$$

It now follows from $(*)$ that $a=2 g$ and $b=2$, as claimed.

Finally, we apply a theorem of Morgan, Szabo, and Taubes to calculate $\mathrm{SW}_{Z_{K}}(k)$. Since $k \cdot \Sigma^{\prime}=2 g$, [MST] applies to give

$$
\mathrm{SW}_{Z_{K}}(k)=\sum \mathrm{SW}_{Z_{K}}(k+2[R])= \pm \mathrm{SW}_{X_{K}}(2 g T) \cdot \mathrm{SW}_{Y}(2 g F)
$$

where the the sum is taken for all distinct classes $k+2[R]$ for $R$ a rim torus. Thus the first equality follows from Lemma 3.1 which shows that each $[R]=0$ in $H_{2}\left(Z_{K} ; \mathbf{Z}\right)$. Now $Y$ is a symplectic manifold with $c_{1}(Y)=-2 g F$; 
so $[\mathbf{T}]$ implies that $\mathrm{SW}_{Y}(2 g F)= \pm 1$. Since we are assuming that $g=d$, [FS2] implies that $\mathrm{SW}_{X_{K}}(2 g T)=a_{d}$, and this completes the proof.

We remark that in case the Alexander polynomial of the knot $K$ does not have maximal degree, the above proof shows that $\mathcal{S} \mathcal{W}_{Z_{K}}=0$; this provides potential examples of simply connected irreducible 4-manifolds with trivial Seiberg-Witten invariants.

Corollary 3.3. Let $K$ be a knot in $S^{3}$ whose Alexander polynomial is not monic and has maximal degree. Then $Z_{K}$ is a nonsymplectic 4-manifold with one basic class.

Proof. The hypothesis implies that the only nontrivial Seiberg-Witten invariant of $Z_{K}$ has value $\pm a_{d} \neq \pm 1$; so $[\mathbf{T}]$ implies that $Z_{K}$ has no symplectic structure. Since $Z_{K}$ contains an embedded sphere of self-intersection -2 , neither does $Z_{K}$ with its opposite orientation admit a symplectic structure.

We close with a comment concerning the geography of our construction. If the genus of $K$ is $g$ then $c\left(Z_{K}\right) \equiv(3 \operatorname{sign}+2 \mathrm{e})\left(Z_{K}\right)=8 g$ and $\chi\left(Z_{K}\right) \equiv$ $\frac{b^{+}+1}{2}\left(Z_{K}\right)=g+2$; so all these manifolds lie on the line of slope 8 passing through $(2,0)=(\chi(K 3), c(K 3))$ in the $(\chi, c)$-plane. We could similarly perform our construction starting with $X=E(2 n)$, the minimal elliptic surface without multiple fibers and with $\chi=2 n$. We then obtain the same result for the lattice points $(3 n+g-1,8(g+n-1))$, i.e., for the lattice points on the line of slope 8 through $(2 n, 0)=(\chi(E(2 n)), c(E(2 n)))$.

\section{References}

[FS1] R. Fintushel and R. Stern, Immersed spheres in 4-manifolds and the immersed Thom conjecture, Turkish J. Math., 19 (1995), 145-157.

[FS2] R. Fintushel and R. Stern, Knots, links, and 4-manifolds, to appear in Invent. Math..

[G] R. Gompf, A new construction of symplectic manifolds, Ann. Math., 142 (1995), 527-595.

[MST] J. Morgan, Z. Szabo and C. Taubes, A product formula for the Seiberg-Witten invariants and the generalized Thom conjecture, Jour. Diff. Geom., 44 (1996), 706-788.

[S1] Z. Szabo, Simply-connected irreducible 4-manifolds with no symplectic structures, preprint.

[S2] Z. Szabo, Exotic 4-manifolds with $b_{2}^{+}=1$, Math. Research Letters, 3 (1996), 731741.

[T] C. Taubes, The Seiberg-Witten invariants and symplectic forms, Math. Res. Letters, 1 (1994), 809-822.

[Th] W. Thurston, Some simple examples of symplectic manifolds, Proc. Amer. Math. Soc., 55 (1976), 467-468. 
[W] E. Witten, Monopoles and four-manifolds, Math. Res. Letters, 1 (1994), 769-796.

Received May 10, 1998 and revised December 15, 1998. The first author was partially supported NSF Grant DMS9704927 and the second author by NSF Grant DMS9626330.

Michigan State University

East Lansing, Michigan 48824

E-mail address: ronfint@math.msu.edu

University of CALifornia

IRvine, CALIFornia 92697

E-mail address: rstern@math.uci.edu 